\title{
LA RECONNAISSANCE PUBLIQUE DES DIFFÉRENCES ET DE LA JUSTICE SCOLAIRE : DE LA SOCIOLOGIE CRITIQUE À LA SOCIOLOGIE DE LA CRITIQUE José Manuel Resende
}

De Boeck Supérieur | Education et sociétés »

2005/1 n 15 | pages 137 à 152

ISSN 1373-847X

ISBN 2-8041-4915-3

Article disponible en ligne à l'adresse :

https://www.cairn.info/revue-education-et-societes-2005-1-page-137.htm

Distribution électronique Cairn.info pour De Boeck Supérieur.

(C) De Boeck Supérieur. Tous droits réservés pour tous pays.

La reproduction ou représentation de cet article, notamment par photocopie, n'est autorisée que dans les limites des conditions générales d'utilisation du site ou, le cas échéant, des conditions générales de la licence souscrite par votre établissement. Toute autre reproduction ou représentation, en tout ou partie, sous quelque forme et de quelque manière que ce soit, est interdite sauf accord préalable et écrit de l'éditeur, en dehors des cas prévus par la législation en vigueur en France. Il est précisé que son stockage dans une base de données est également interdit. 


\title{
La reconnaissance publique des différences et de la justice \\ ૧૯ scolaire : de la sociologie critique à la sociologie de la critique
}

\section{De Boeck Université | Education et sociétés}

\author{
2005/1 - no 15 \\ pages 137 à 152 \\ ISSN 1373-847X
}

Article disponible en ligne à l'adresse:

http://www.cairn.info/revue-education-et-societes-2005-1-page-137.htm

\section{Pour citer cet article :}

"La reconnaissance publique des différences et de la justice

0 scolaire : de la sociologie critique à la sociologie de la critique", Education et sociétés, 2005/1 no 15, p. 137-152.

Distribution électronique Cairn.info pour De Boeck Université.

(c) De Boeck Université. Tous droits réservés pour tous pays.

La reproduction ou représentation de cet article, notamment par photocopie, n'est autorisée que dans les limites des conditions générales d'utilisation du site ou, le cas échéant, des conditions générales de la licence souscrite par votre établissement. Toute autre reproduction ou représentation, en tout ou partie, sous quelque forme et de quelque manière que ce soit, est interdite sauf accord préalable et écrit de l'éditeur, en dehors des cas prévus par la législation en vigueur en France. II est précisé que son stockage dans une base de données est également interdit. 


\title{
La reconnaissance publique des différences et de la justice scolaire : de la sociologie critique à la sociologie de la critique ${ }^{1}$
}

\author{
José MANUEL RESENDE \\ Departamento de Sociologia \\ Faculdade de Ciências Sociais e Humanas, Universidade Nova de Lisboa \\ Unité d'investigation Forum Sociológico - Centro de Estudos \\ josemresende@mail.telepac.pt
}

\section{La construction du projet de la modernité en Europe occidentale et la forme scolaire moderne : brèves questions sur une problématique plus vaste}

T e concept de modernité, selon le point de vue d'Anthony Giddens (1992, L Giddens, Beck \& Lash 2000) et celui de Peter Wagner (1996), a été introduit récemment dans les réflexions sur l'éducation au Portugal (Resende 2001a,b ; Vieira 2001). Les racines historiques des principales caractéristiques de la modernité en Europe occidentale doivent servir, en raison de leur valeur heuristique, à nourrir l'interprétation sociologique de la société contemporaine et du temps présent.

L'idée de la contemporanéité de la définition de modernité est pour Martuccelli "le plus petit dénominateur commun" $(1999,10)$ du concept. Dès lors, il

I Cet article résume une communication, "La Construction de la démocratisation scolaire : perspectives historiques et sociales", présentée lors de la Conférence "La démocratisation scolaire : intentions et appropriations” organisée par Maria Manuel Vieira, Joaquim António Pintassilgo et Maria Benedita Portugal à la Faculté des Sciences de I'Université de Lisbonne le I6 octobre 2002. Je remercie Maria Manuel Vieira pour cette invitation. Les présentes réflexions ont une double genèse. D'un côté de longues discussions avec Maria Manuel Vieira, déjà publiées (Resende \& Vieira 2000, 2002) et de l'autre, les réflexions conduites pour mon doctorat (Resende $200 \mathrm{Ia}$ ). Je remercie particulièrement Leonor Sampaio qui a adapté pour Éducation et Sociétés ce texte déjà publié en portugais in Vieira M.-M., Pintassilgo J. \& Portugal e Melo B. (coords) 2002 Actas do Colóquio "Democratização escolar: intenções e apropriações”, Lisboa, Centro de Investigação em Educação da Faculdade de Ciências da Universidade de Lisboa, I43-I72. 
n'est pas inapproprié d'en faire l'axe central de tous les questionnements sur le temps présent. En ce sens, une analyse historique et sociologique de la modernité (Wagner 1996) rend possible la compréhension et l'interprétation des ambiguïtés qui apparaissent dans divers discours et pratiques, soit résultant du travail institutionnel et formel, soit résultant des activités simples se produisant au gré des rencontres quotidiennes et informelles. En vérité, la configuration discursive qui s'est profilée à partir du XVIIe siècle, et qui s'est objectivée dans des événements politiques, économiques et sociaux remarquables -la Révolution française, la Révolution démocratique américaine et la Révolution industrielleoblige à prendre en compte dans l'analyse sociologique deux dimensions: la liberté et la discipline.

Selon Nisbet, “ce qui caractérise le libéralisme, c'est la foi en l'individu et l'affirmation de ses droits politiques, civiques et, plus tard, sociaux. L'autonomie de l'individu présente la même valeur pour le libéral que la tradition pour le conservateur et l'utilisation du pouvoir pour le radical" (Nisbet 1993). C'est, dès la fondation du projet libéral que la liberté, l'autonomie et l'autoréalisation de la condition humaine moderne trouvent une résonance dans les discours et les représentations produites par des penseurs qui critiquaient l'ordre social de l'Ancien Régime.

Les tensions et les ambiguïtés produites par le caractère irréductible des idéaux de liberté et de discipline traversent tous les processus servant la construction historique du projet de la modernité, qui ne reste pas exclusivement accrochée à la période de la modernité libérale stricte. La contestation politique et sociale des dernières décennies du XIX e siècle accélère, d'un côté, la construction d'autres formes d'organisation pour les transactions se faisant dans les multiples chaînes d'interaction, et, d'un autre côté, elle renforce la nécessité de l'existence des institutions étatiques pour la construction de réseaux de pouvoir, ajustés maintenant à un grand nombre de conventions qui essaient de concilier les intérêts de classe différentes.

La pluralité et l'ouverture des pratiques et des identités des individus modernes annoncées à travers les discours postmodernes ne sont pas seulement le résultat direct du questionnement sur les possibilités de concrétiser le projet de la modernité, mais aussi du degré de compréhension qu'en ont les citoyens concernés. Le travail de réflexivité réalisé par les acteurs modernes sur leur propre corps a été facilité et rendu possible à travers la médiation des sciences sociales (Giddens 1995). La connaissance de soi-même et la reconnaissance par les autres de l'authenticité de ses caractéristiques culturelles propres sont des thématiques apparaissant dans des discours destinés à faire reconnaître publiquement des différences (Taylor 1998). Des différences qui résultent soit des caractéristiques d'identification de sa propre identité personnelle, soit des 
caractéristiques identitaires d'un groupe socialement désigné à partir d'un attribut comme le genre, l'orientation sexuelle, l'ethnie ou la confession religieuse.

Comment la forme scolaire moderne intervient-elle dans ce questionnement généralisé sur les différences, en particulier sociales et culturelles, et sur le caractère injuste de la non-reconnaissance publique? Avant de répondre, il convient de formuler quelques idées sur les relations qui peuvent être établies entre le projet de la modernité et la forme scolaire moderne.

En réalité, la forme scolaire moderne peut être comprise comme une institution sociale qui participe effectivement à l'effort de diffusion des idéaux qui donnent forme au projet de la modernité. Telle que d'autres institutions sociales, la forme scolaire moderne présente, tout au long de son histoire, des configurations sociétaires fondées sur "des ensembles relativement durables de règles et de ressources, sur lesquelles les gens peuvent s'appuyer dans leur action" quotidienne (Wagner 1996, 47).

À travers cette activité pratique continue, ces acteurs travaillent pour la construction des conventions (Wagner 1996, Livet 1993), qui, basées principalement sur les ressources et les règles déjà existantes, contribuent aussi à la transformation de ces mêmes fondations. En ce sens, les règles, les ressources et les conventions institutionnalisées par l'école sont des répertoires qui habilitent les individus modernes, mais qui créent simultanément des limites aux actions humaines, posant des contraintes à travers un travail de subjectivation réalisé par un projet de socialisation singulier (Queiroz 1995).

La singularité du projet de socialisation tient dans sa différence avec le type d'apprentissage assuré dans la famille. Le travail pédagogique personnalisé de la socialisation familiale est remplacé par un travail impersonnel de mise en forme des conduites réalisées par un ou plusieurs professeurs, qui, dans la salle de classe, se trouvent devant un groupe d'élèves.

Le processus d'autorégulation des postures corporelles ne se fait plus selon une routine mécanique, sur simple imitation, par osmose. La reconnaissance de la valeur d'un comportement ajusté aux canons de l'école se fait en relation avec un corps de règles établies, et l'évaluation de son amélioration s'adosse à un modèle de comportement explicité par le code de conduites scolairement légitimes.

L'invention de la relation pédagogique selon la forme scolaire moderne lui donne une singularité notable soumise aux altérations successives résultant des réformes imposées par un État centralisé et dont le travail est lui aussi fondé sur des règles impersonnelles soumises à la logique d'une rationalité bureaucratique (Vincent 1994, Queiroz 1995).

Au fil du temps et dans différents contextes, l'École se transforme en une institution d'État bien que la diffusion de la forme scolaire moderne ne soit pas réalisée selon un plan conçu de manière délibérée. Toutefois, il n’y a aucun 
doute sur la contribution de la forme scolaire moderne à la construction de l'identité nationale, à la célébration de l'esprit d'une culture urbanisée, ainsi qu'à l'intégration dans le cadre des valeurs qui sont celles de la modernité (Vincent 1994, Queiroz 1995).

D'autre part, la scolarisation moderne a produit encore deux effets importants - la codification et la formalisation- qui graduellement gagnent tous les groupes sociaux, à travers la portée macrosociale des politiques d'éducation fondées sur des principes d'égalité des chances et de l'allongement de l'obligation scolaire. Les efforts de codification des relations pédagogiques -objectivés, par exemple, à travers les épreuves servant à établir le scolaire- résultent d'un travail accompli par l'organisation scolaire tout au long de l'année, défini par des règles établies par l'appareil juridictionnel d'État (Abbott 1988) et donnant lieu à des certifications attribuées par l'institution scolaire.

La naturalisation de ces deux effets - de codification et de formalisation-, au fil de l'installation graduelle et non linéaire de la forme scolaire moderne, ne soumet pas totalement les esprits critiques que cette institution prétend aussi former grâce au travail cognitif qu'elle réalise et généralise graduellement. En effet, dès sa fondation, l'école moderne œuvre à combattre l'obscurantisme et l'ignorance et, en cela, elle est une alliée de la pensée des Lumières du XVIIIe siècle.

Le primat de la raison et des savoirs fondés sur une logique rationnelle se combine au besoin que l'école éprouve de promouvoir la discipline comme des valeurs indispensables autant pour l'acquisition des connaissances que pour la consolidation des mœurs civilisées. Dans ce sens, le double apprentissage, cognitif et civique, effectué par la scolarisation rend possible l'inscription dans une même institution des deux faces de la modernité identifiées par Wagner (1996), qui se trouvent intégrées dans les représentations qui sont celles de l'élite moderne et éclairée (Eisenstadt 1991) : la liberté et la discipline.

\section{De la reconnaissance publique et politique des différences culturelles aux mouvements de résistance pédagogique : les dénonciations de la domination culturelle de la forme scolaire moderne transnationale - autre forme d'interprétation de la sociologie critique}

Stoer \& Araújo (1991) sont arrivés à des conclusions identiques à celles de Bourdieu et son équipe (Bourdieu 1965, 1970) à propos des fonctions sociales assurées par l'École et par les enseignants dans les sociétés modernes. Avec un regard élargi, ces deux auteurs se montrent critiques à l'égard de l'intervention des organismes internationaux dans les décisions de politiques éducatives nationales. Le développement du système d'enseignement dans la ligne d'un modèle unique, ainsi que l'obligation scolaire imposée comme mesure à faire respecter 
universellement par toute la population portugaise, indépendamment des localisations géographiques (ville-campagne), des appartenances de classe (l'expansion du salariat à la campagne), des origines culturelles liées à des caractéristiques ethniques, ont constitué une forme de médiation nocive exercée par les organismes internationaux (OCDE, Banque Mondiale, FMI, etc.), justifiant l'imposition de telles mesures comme compensation de l'appui financier fourni au Portugal.

L'effet pervers de toutes ces mesures "a été de régler politiquement la gestion de savoir qui est et qui n'est pas salarié" à travers "l'introduction d'un système d'éducation commun, se substituant aux formes familiales de formation professionnelle et de socialisation", "sur base d'une durée bien définie pour tous de l'obligation scolaire” (Stoer \& Araújo 1991, 209). Cela a écarté nombre de jeunes du marché du travail.

Cette importation de modèles étrangers au Portugal a eu comme conséquence la conservation et l'expansion de la logique capitaliste, interprétée non seulement comme un système d'exploitation des relations entre les classes asymétriquement situées dans la structure sociale (dans les marchés de la propriété patrimoniale privée, du travail, financier, culturel, etc.), mais aussi comme un système de régulation politique et idéologique généralisant certains mécanismes de socialisation, notamment la socialisation scolaire qui a permis de promouvoir au sein de la population scolaire des attitudes de conformité civique grâce à l'importation de la "rationalité technique" propre aux espaces économiques (Stoer \& Araújo 1991, 210 ; Aronowitz \& Giroux 1992). Ainsi, les systèmes scolaires seraient prisonniers des logiques économique et technique constamment réinventées au long de l'histoire du capitalisme.

Selon quelques auteurs (Enguita 1996), l'effet culturel dévastateur déclenché par la scolarisation obligatoire se trouverait directement associé à une justification arbitraire de la nécessité pour l'État libéral et démocratique d'étendre à tous les groupes sociaux le principe de l'égalité des chances grâce à l'obligation scolaire. Mais cette obligation n'est pas néfaste seulement pour la préservation de l'authenticité culturelle des minorités ethniques. Le modèle organisationnel de l'école elle-même, imposé d'une façon uniforme par l'État centralisateur qui cherche à obtenir une intégration et une soumission de toutes les populations résidant sur un territoire, indépendamment de tous leurs attributs sociaux et culturels spécifiques, ne semble pas être le plus adéquat en ce sens. En réalité, comme Enguita nous l'explique, l'État moderne, démocratique et libéral n'a pas le droit d'étendre à tous la prérogative de jouissance d'un bien commun (comme la fréquentation de l'école pendant un nombre d'années bien défini). Cela est particulièrement vrai en ce qui concerne les minorités ethniques et culturelles dont les membres manifestent des conduites inadaptées face aux standards culturels dominants. Ainsi en va-t-il des enfants d'origine gitane qui se présentent 
dans les écoles sans y avoir été préparés et qui ont du mal à y réaliser des activités qui leur semblent terriblement monotones et qui n'ont pas de sens pour leur famille (Enguita 1996, 16). L'inadéquation entre le modèle culturel de l'école et les normes culturelles de ces minorités ethniques (envisagées ici comme des groupes homogènes, avec des intérêts communs, qui se comportent selon des régimes d'action si singuliers qu'ils provoquent leur exclusion sociale et culturelle) se traduit par l'impossibilité pour ces individus d'être instruits ou socialisés par l'école.

Cette double exclusion transforme le multiculturalisme des sociétés modernes, capitalistes et développées en problème social, reconnu autant dans sa dimension éducative (concernant soit le domaine de l'instruction, soit celui de la socialisation), que dans sa dimension politique (concernant la participation critique et la reconnaissance de leurs singularités culturelles qui doivent être respectées et préservées dans leur authenticité).

Un des buts proclamés par les défenseurs de cette version de la sociologie critique, notamment les défenseurs de la construction d'une théorie critique postmoderne est de contrarier la finalité disciplinaire, hiérarchique et usurpatoire du système scolaire -résultante des ambiguïtés traduites par les discours modernes de la régulation et de la liberté- dues aux interférences néfastes de la rationalité technique originaire de l'économie capitaliste (Santos 1999). Pour contrarier la domination prévisible de cette rationalité technique et bureaucratique, objectivée dans les programmes scolaires, dans les programmes de formation des professeurs, dans l'organisation et le fonctionnement des écoles, les territoires construits par le système éducatif doivent être des espaces sensibles aux sollicitations égalitaires et démocratiques sollicitées par des groupes de citoyens ordinaires, notamment les groupes intellectuellement plus actifs (Nóvoa 1991a, b).

Selon la perspective critique postmoderne (Santos 1999) ou dans l'analyse critique de résistance (Giroux 1986, 1997 ; Aronowitz \& Giroux 1992) ou encore dans une autre version plus circonscrite, maintenant appelée "le champ de re-contextualisation pédagogique” (Stoer 1994, Cortesão \& Stoer 1997, Stoer \& Cortesão 1999), ces auteurs diagnostiquent la crise de la modernité réflexive (Beck, Giddens \& Lash 2000) et infèrent des mesures pratiques pour combattre les injustices et les oppressions sociales et culturelles créées par les procédés de modernisation en action dans toute la planète.

Pour la théorie critique postmoderne appliquée dans l'analyse des mouvements multi-culturalistes de l'espace scolaire, le combat des injustices se fait avec l'intervention de la connaissance, mais d'une connaissance d'une autre nature, distincte du caractère universel de la raison et du cogito divulgué par le projet imaginé de modernité. Dans ce sens, ce nouveau type de connaissance est solidaire avec les autres, particulièrement avec les autres qui sont victimes de l'oppression du capitalisme et de son actuelle version globalisée -la trans-nationalisation du seul modèle économique engendré dans les pays centraux. "La 
solidarité [étant] une forme de connaissance qui s'obtient à travers la reconnaissance de l'autre, l'autre peut seulement être connu en tant que producteur de la connaissance. C'est pourquoi toute la connaissance/émancipation a une vocation multiculturelle" (Santos 1999, 29).

Cependant, cette connaissance/émancipation ne doit pas être soumise au caractère universel de la raison divulgué par le projet imaginé de la modernité. Selon l'auteur, "on ne doit pas oublier que, sous la couverture des valeurs universelles autorisées par la raison, fut imposée la raison d'une 'race', d'un sexe et d'une classe sociale. La question étant comment réaliser un dialogue multiculturel si quelques cultures ont été réduites au silence et leurs formes de voir et de connaître le monde sont devenues imprononçables ?" (Santos 1999, 30).

Cherchant l'inspiration dans le legs théorique de l'École de Frankfurt, cette perspective se détache de la croyance moderniste concernant les capacités illimitées de la raison instrumentale modernisatrice qui envisage de "sauver le monde des chaînes de la superstition, de l'ignorance et de la souffrance" (Giroux 1986, 26). Dans ce sens, la mission de la "vraie" raison envisageait la conversion des êtres humains en individus critiques de façon à permettre leur émancipation face aux contraintes sociales, en particulier face aux modalités de domination des sociétés capitalistes. C'est pour cela que la raison comprend une éthique, de telle sorte que l'autocritique et le questionnement de son propre cadre normatif sont possibles.

Pour que la raison soit opératoire, ce qui veut dire critique et interrogeant le réel, il est indispensable de formaliser une théorie critique "pour jouer avec les standards de conflit et de la contradiction qui existaient dans les différentes sphères culturelles" (Giroux 1986, 62), avec le but de créer "une théorie adéquate de la conscience sociale" (Giroux 1986, 63).

La théorie de base culturaliste et sa ramification dans le domaine de la conscience sociale des individus deviendront positives si elles arrivent à redéfinir des fonctions et des finalités de l'éducation et de la scolarisation moderne. Leur premier combat a été leur contribution à la création de la pédagogie critique fondée sur l'attention portée aux luttes culturelles dans les écoles (Giroux 1986, 100), ou dans le même sens elles ont fait fonctionner dans les écoles "le champ de re-contextualisation pédagogique” (Stoer 1994, Cortesão \& Stoer 1997), (Stoer \& Cortesão 1999). Ce qui était important dorénavant, c'était d'avoir une vision complètement différente sur le curriculum caché, d'ailleurs considéré ici comme une vraie boîte noire non seulement de l'école elle-même mais aussi de toute la société. Dans ce sens, l'analyse du curriculum caché devrait comprendre "toutes les instances idéologiques du procès de scolarisation qui 'silencieusement structurent et reproduisent les présupposés et les pratiques hégémoniques"” (Giroux 1986, 100-101), à la fois celles de nature essentiellement sociale et celles de nature essentiellement culturelle. 
La signification "nouvelle" attribuée à cette boîte noire a rendu possible la constitution d'un autre topique de la théorie critique et de résistance dans l'éducation : du "souci unilatéral autour de la reproduction culturelle" on est passé "au souci autour de l'intervention culturelle et de l'action sociale" (Giroux 1986, 101). La consécration de l'intervention sociale et culturelle a transformé l'école en espace de contradictions et de luttes, reflet direct de la complexité macrostructurelle. Étant un espace de luttes, la finalité de cette instance était de s'engager pleinement dans la lutte pour l'émancipation.

Pour cela, l'école et son support théorique devaient constituer le vecteur indispensable au succès de ce combat : la formulation de la pédagogie critique ou la création du "champ de re-contextualisation pédagogique" (Stoer 1994, Cortesão \& Stoer 1997, Stoer \& Cortesão 1999). La première s'appuyait sur un "discours qui [clarifiait] les conditions idéologiques et matérielles nécessaires à la promotion des modes critiques de scolarisation et des modes alternatifs d'éducation pour la classe ouvrière et pour d'autres groupes qui servent l'impact de l'oppression politique et économique" (Giroux 1986, 304), en particulier les minorités dites ethniques. Pour le cadre de la re-contextualisation pédagogique, l'important est d'utiliser les stratégies d'éducation, dans le domaine d'une déconstruction effectuée à partir d'une recherche-action, dans le sens d'identifier les modalités adéquates pour combattre la "logique de la réalisation méritocratique", une fois que le développement de sa rationalité pratique "se traduit dans des obstacles multiples à la construction du professeur inter/multiculturel" (Stoer 1994, 20).

En faisant dépendre la prise de conscience du professeur du démontage réalisé par la sociologie critique et de la résistance de la relation linéaire établie entre l'État et ce corps de professionnels, ses défenseurs produisent un point de vue sur les enseignants adossé à une perspective substantialiste que la connaissance ordinaire et la connaissance scientifique (Boltanski 1970) tendent à construire et à solidifier à travers les débats autour des concepts de profession, d'ethnie ou encore "des expectatives culturelles et cognitives". Tous ces concepts sont conçus comme des identités homogènes ou comme des groupes dont les membres partagent une identité organique et fonctionnellement équilibrée, une fois qu'ils semblent communier dans les mêmes intérêts. Pour se défendre, ils développent des stratégies d'émancipation à sens unique et dont l'accord, toujours constant, est ancré sur la réalité naturalisée des choses et du monde qui les entoure. Plutôt que de suivre cette vision dualiste du jugement des professeurs, notre perspective d'analyse s'approche de la sociologie de la critique qui présente un modèle composite pour l'interprétation des formes de jugement et des actions de tous les acteurs (profanes et savantes). 


\section{Les représentations de la forme scolaire moderne dans le questionnement produit par la sociologie de la critique : du jugement critique à l'épreuve de la justice scolaire}

D our traiter de la question de la justice scolaire dans les sociétés du début du XXIe siècle, il faut déplacer l'attention vers le local, comme le dit Derouet : "l'idée du projet d'établissement puis l'extension des pratiques de contrat répondent à la nécessité de trouver des techniques de réaccord, dans un contexte d'incertitude sur la légitimité et les buts de l'éducation" (Derouet 1992, 78).

La question est à mettre en relation avec l'émergence d'un sentiment d'impuissance généralisée à enrayer définitivement les inégalités scolaires et sociales résultantes de la massification scolaire (dans les pays modernes). Cela ne signifie pas que le combat pour une école plus juste ait été mis en cause. Certes, il y a eu un déplacement du regard politique vers les engagements locaux, correspondant, d'ailleurs, aux intentions manifestées par les spécialistes et les chercheurs du domaine scientifique.

L'intérêt pour le local correspond à un passage du général au particulier. D'une certaine façon, après la révolution des Lumières, c'était à l'État qu'il revenait de s'acquitter du travail de libération de la société civile face aux contraintes sociales et politiques créées par le pouvoir sous l'Ancien Régime. À travers l'École, l'État “disposait d'un puissant instrument de pilotage de la société civile" (Derouet 1992, 276). De cette façon, il bénéficiait des résultats du travail de socialisation scolaire. Celle-ci inculquait des valeurs éthiques et morales et contribuait à la distribution/redistribution des positions dans la structure sociale.

La question de la justice scolaire et sociale ne se résolvant pas à l'échelle macrosociale, la décentralisation territoriale en faisant glisser l'intérêt des recherches vers le local renouvelle le débat scientifique. Autrement dit, on se demande maintenant si l'objet, envisagé à partir de l'École en tant qu'espace singulier, ne met pas en cause les contraintes face à la légitimité, soit en relation avec les régimes d'action, soit en relation avec les modèles de justification (Boltanski \& Thévenot 1991, Boltanski 2001) fondés d'un côté sur le principe de l'ordre et, de l'autre, sur le principe de commune humanité. C'est à la théorie sociologique de résoudre ce problème.

Dans le cas de disputes résultant de l'identification de situations d'injustice, les différents acteurs individuels et collectifs présents dans les établissements d'enseignement sont invités à se prononcer sur des expériences vécues comme injustes. Au cours de cette confrontation, simultanément pratique et discursive, les élèves, les professeurs et les parents, formulent des jugements sur les injustices scolaires. Dans cette optique, le modèle d'action justifiable donne aux 
acteurs la capacité de réaliser un travail de coordination des activités humaines à travers des tentatives de rapprochement entre individus qui sont confrontés à un problème : la crise du système scolaire, le malaise des enseignants, l'interférence abusive des parents dans leur travail, la rigueur excessive des classements scolaires ou encore les doléances sur la dévalorisation du travail des professeurs. Ces différentes situations constituent des moments privilégiés pour préciser les différents principes de justification mobilisés par les acteurs concernés. Dans l'épreuve de justification, le langage met les choses en relief. Tout le travail d'argumentation développé par les acteurs étant d'une extrême importance et objectivant leurs capacités de réalisation.

Nous comprenons à travers des actions collectives justifiables, les actions "qui supposent des exigences fortes de contrôle d'un jugement commun", bien qu'un tel jugement ne puisse pas "reposer sur les hypothèses d'accord et de compréhension commune que véhiculent les notions de norme sociale ou de valeur. Il faut partir des modalités de traitement des attentes non satisfaites, visibles dans les disputes, les critiques ou les expressions d'injustice" (Thévenot 1993, 277, Boltanski 1993).

Et nous croyons que c'est ce qui arrive quand on met en cause les injustices commises par des modèles de scolarisation dont les résultats ne s'ajustent ni aux attentes des groupes d'élèves et de leurs parents ni à celles des groupes d'enseignants. Pour les premiers, les pratiques scolaires -objectivées, par exemple, dans les actes de jugement produits par certains membres du corps enseignant- sont excessivement sélectives, mais ils contestent surtout les modalités d'évaluation et les critères établis dans les grilles de classification adoptées. Pour les seconds, l'entrée massive des publics socialement et culturellement hétérogènes, renforcée par la reconnaissance du manque de conditions de travail adaptées ou le manque d'une formation professionnelle adéquate pour répondre a ces nouveaux défis, crée chez quelques-uns un sentiment de malaise, résultat d'une impression étrange (Bauman 1998) provoquée par une modification de la morphologie du corps des élèves.

La sociologie de la critique, en déplaçant l'analyse vers des situations scolaires concrètes, construites par les acteurs qui y sont engagés, a fini par se distancier de la sociologie critique qui, indépendamment de ses variantes conceptuelles, maintient son investigation de haut en bas, ce qui signifie qu'elle élit comme son objet fondamental l'architecture générale du système et ses multiples relations avec la société, l'économie et le monde. De la perspective situationnelle où les acteurs se trouvent en interactions fréquentes, la sociologie de la critique élabore des postulats analytiques sur les régimes d'action organisés par leurs protagonistes à travers l'utilisation sociale de leur capacité cognitive, politique et technique de représentation des relations et des objets impliqués, pour tracer les contours théoriques éventuels de la façon dont la représentation de la modernité contemporaine se déploie. 


\section{La dévaluation du travail scolaire dans la construction d'une école plus juste?}

\section{Du caractère universel et cosmopolite de l'École à la reconnaissance de la singularité des cultures locales}

Les discours et les représentations sociales liés à la crise scolaire s'en prennent toujours aujourd'hui aux activités de cette institution. La critique principale adressée à l'École tient dans le fait qu'elle n'aurait pas encore accompli la mission historique que la modernité lui a assignée dès le XVIIIe siècle.

En vérité, depuis la genèse de la forme scolaire moderne, ses concepteurs y ont mis des espoirs énormes, particulièrement pour la diffusion des idées des Lumières, soucieuses de la transmission des connaissances scientifiques, littéraires et techniques et de la consolidation d'un ethos laïc en relation avec l'extension de la "civilisation des mœurs". Ces codes ont renvoyé à des normes morales et éthiques qui correspondaient d'abord aux principes normatifs propres à l'esprit bourgeois et urbain. Mais, surtout à partir de la fin du XIXe siècle, ils semblent s'être adaptés aux principes normatifs inscrits dans la machinerie juridictionnelle des États modernes (Abbott 1988), lesquels ont fait de la scolarisation universelle le moyen le plus efficace pour promouvoir l'intégration politique et sociale des citoyens, pour consolider l'identité nationale, la cohésion, le progrès civilisateur et le bien-être économique.

Les dispositifs juridiques généraux -les fonctions et les finalités de la scolarisation-, l'organisation des espaces et des temps dévolus à l'éducation et à l'instruction, la définition des disciplines et des programmes scolaires, l'élaboration du statut de l'élève et du professeur, etc., constituent quelques-uns des outils normatifs au service de l'État moderne qui lui ont permis de s'approprier sur tout son territoire le monopole de la violence légitime. En ce sens, l'École contribue à ancrer le principe d'une discipline consentie ou encore la socialisation scolaire incite les citoyens modernes à accepter l'existence de réseaux de pouvoir et de conventions fondées sur un ordre justifié mais qui peut être contesté dans les limites prévues par la loi (Wagner 1996, Boltanski \& Thévenot 1991, Boltanski 2001).

Grâce à la consécration de l'universalité des savoirs et des connaissances apprises à l'école au cours d'une période de temps variable, l'École participe directement à la construction de l'idéal d'autoréalisation humaine et civique, moyen indispensable pour l'arrachement des citoyens modernes à d'autres influences ou dépendances, soit locales, soit religieuses. Cependant, les jugements critiques développés sur l'activité scolaire ont presque toujours des incidences sur deux domaines liés aux deux dimensions fondatrices du projet imaginé de la modernité : le principe de liberté et celui de discipline. 
En résumé, les analyses multiculturalistes relevant d'une sociologie critique interprètent la crise scolaire à partir du modèle de justification civique (Boltanski \& Thévenot 1991, Boltanski 2001). De fait, les investissements critiques énoncés dans cet article, portent surtout sur les difficultés ressenties par les sciences de l'éducation face au développement d'une pensée critique dans les présupposés nuancés tant par la philosophie de la science moderne que par la sociologie de la connaissance scientifique moderne (Santos 1999). La seule façon de mobiliser les arguments civiques du côté des intervenants plus proches de l'univers scolaire -des professeurs aux politiciens, des parents aux techniciens et aux spécialistes de l'éducation- c'est à travers la déconstruction des présuppositions catéchisantes qui sont à la base des analyses réalisées par la science critique moderne.

Seule la critique effectuée par la logique de la connaissance multiculturelle et par la voie de sa traduction dans le topique de l'herméneutique fondée sur la reconnaissance de l'intelligibilité des différences (Santos 1999) peut combattre efficacement la rationalité instrumentale implantée soit dans les jugements scientifiques, soit dans le travail pédagogique pratiqué dans les différentes écoles. D'un autre côté, les propriétés d'interprétation du type de connaissance, appuyées par la version culturaliste de la sociologie critique, exigent aussi une forte relevance théorique pour leurs promoteurs : la connaissance des contextes et des conditions sous lesquelles cette connaissance multiculturelle et herméneutique est créée.

À travers cette reconnaissance, non seulement il sera possible d'éviter les effets nocifs des explications résultant de l'apparente nature universelle de la connaissance scientifique, mais il sera aussi possible d'attribuer un crédit équivalent aux connaissances multiculturelles singulières liées aux groupes culturels minoritaires, d'origine ethnique, sexuelle ou sociale. L'application de ces principes dans "le champ de la re-contextualisation pédagogique" telle qu'elle est discutée par ses défenseurs (Stoer 1994, Cortesão \& Stoer 1997, Stoer \& Cortesão 1999) crée les conditions idéales pour valoriser les savoirs et les pratiques des élèves porteurs des cultures dominées.

Le nouveau sens attribué aux contextes d'action pédagogique inter/multiculturelle donne la possibilité d'associer la critique aux présupposés culturels dominants sur lesquels les curricula et les programmes des disciplines s'appuient ; à travers ce travail de déconstruction ce nouveau sens évalue la pertinence des mouvements politiques de résistance culturelle et le rôle politique de leurs mandataires. La fragmentation, la pluralité et la non-universalité des savoirs et des connaissances scientifiques transmises par l'école justifient l'importance des processus d'autonomisation des établissements d'enseignement, des stratégies de négociation entre les professeurs et les élèves appartenant à des minorités ethniques. Autant de facteurs décisifs pour mettre en cause les principes normatifs de 
l'encadrement idéologique explicité par les défenseurs des classifications scolaires, en particulier les défenseurs des procédés d'évaluation fondés sur les mérites démontrés dans les épreuves effectuées par les individus scolarisés.

La seule épreuve scolaire acceptée par les multiculturalistes (Enguita 1996) concerne maintenant l'authenticité des cultures révélées par les élèves et fondée sur leurs styles de vie. Néanmoins, la validité de leur appréciation exige un changement radical dans l'organisation et dans la forme du fonctionnement des écoles. Originaires de contextes culturels et/ou nationaux où l'État n'existe pas ou n'est pas présent sur tout le territoire national, les styles de vie, les identités personnelles, culturelles et sociales ainsi que les pratiques de ces élèves ne s'adaptent pas aux exigences de discipline et d'ordre justifié sur lesquelles s'appuient les réseaux de pouvoir et de transaction de la forme scolaire moderne.

La revalorisation scolaire des différentes dimensions culturelles, singulières et avérées, des groupes minoritaires constituent le milieu privilégié par les multiculturalistes pour la reconnaissance de leur authenticité. À travers elle, leurs mandataires voient garantie leur survivance culturelle, renforcée maintenant par la consécration de leur identité primaire : l'identité ethnique, sexuelle, de genre, etc.

Dans ce sens, le fonctionnement correct du "champ de re-contextualisation pédagogique" crée les conditions objectives et subjectives pour que les enseignants déconstruisent les présupposés idéologiques, le pouvoir et la domination symbolique construits patiemment par les classes dominantes (Bourdieu 1989). L'explicitation des principes normatifs liés au pouvoir de classification culturelle développé par les membres des cultures dominantes joue en faveur des groupes culturellement dominés, à travers des révélations opérées par les intellectuels enseignants. D'ailleurs les fonctions de ce groupe professionnel d'avantgarde constituent une des bases les plus importantes pour tous les groupes dominés, une fois que leur travail de déconstruction permet de cautionner le mouvement de contre-hégémonie défendu par les utopies de résistance politique et culturelle.

De l'autre côté, ce travail critique de déconstruction de la légitimité symbolique qui donne forme aux cultures dominantes, transforme l'École en une espèce de dernière réserve de préservation de la singularité et d'authenticité culturelle des minorités en général, ethniques en particulier. Et les professeurs sont choisis comme leurs tuteurs ou leurs protecteurs pour tout ce qui concerne les injustices commises par la forme scolaire moderne.

Sans observer, sans entendre et sans identifier les attentes explicitées par ces individus sur le temps qui passe (Serres 1996), mais surtout sans construire les régimes d'action objectivés par ces auteurs à partir de leurs dispositions critiques, discursives ou pratiques, les multiculturalistes se présentent comme les légitimes porte-parole publics et politiques de leurs aspirations concrètes. Dans toute l'amplitude de leur action de compassion et de bonté, au sens de Boltanski 
(1990), les défenseurs des cultures minoritaires, au nom de la construction d'une école plus juste, décident de critiquer la forme scolaire moderne, ses épreuves, la nature universelle et cosmopolite des savoirs qu'elle transmet.

Que faire si les membres des groupes sociaux et culturellement minoritaires trouvent des sens concrets coupés des domaines cognitifs et de socialisation développés par la forme scolaire moderne? Il faudrait alors contrarier la production de ces attentes plurielles -d'intégration, d'exaltation, de croisement culturel, etc.-, en dévaluant le travail pédagogique et scientifique et les principes normatifs qui, à la fois, orientent, limitent les actions de tous les groupes scolaires, les habilitent, contribuant ainsi à leur critique à l'école ou dans d'autres conjonctures quotidiennes?

Peut-être l'alternative proposée par Derouet, le débat autour des injustices provoquées par la forme scolaire moderne, doit-il être mis en équation à partir des questions suivantes: "de quoi le petit homme a-t-il besoin pour développer son humanité ? Quelles sont les compétences qui sont nécessaires pour être un membre actif de la cité ? Et comment assurer la transmission culturelle entre les générations tout en donnant des chances égales à tous ?" (Derouet 2001, 29).

\section{Bibliographie}

ABBOTT A. 1998 The System of Professions. An Essay on the Division of expert Labor, Chicago, London, The University of Chicago Press

ARONOWITZ S. \& GIROUX H. 1992 Educação radical e intelectuais transformadores, in Esteves A. E J., Stoer S. R. (org.) A Sociologia na Escola - Professores, Educação e Desenvolvimento, Porto, Edições Afrontamento, 145-166

BAUMAN Z. 1998 O Mal-Estar da Pós-Modernidade, Rio de Janeiro, Jorge Zahar Lda

BECK U., GIDDENS A. \& LASH S. 2000 Modernização Reflexiva. Política, Tradição e Estética no Mundo Moderno, Oeiras, Celta

BOLTANSKI L. 1970 "Taxonomies populaires, taxonomies savantes: les objets de consommation et leur classement", Revue Française de Sociologie, XI, 34-44

BOLTANSKI L. \& THÉVENOT L. 1991 De la justification. Les économies de la grandeur, Paris, Gallimard

BOLTANSKI L. 1990 L'amour et la justice comme compétences. Trois essais de sociologie de l'action, Paris, Métailié

BOLTANSKI L. 1993 Dissémination ou abandon : la dispute entre amour et justice. L'hypothèse d'une pluralité de régimes d'action, in Ladrière P., Pharo P., Quéré L. (coord.) La théorie de l'action. Le sujet pratique en débat, Paris, CNRS Éditions, 235-259

BOLTANSKI L. 2001 "A moral da rede ? Críticas e justificações nas recentes evoluções do capitalismo", Forum Sociológico, 5/6 (nova série), 13-35

BOURDIEU P., PASSERON J.-C. \& SAINT-MARTIN M. de 1965 Les étudiants et la langue d'enseignement, in Rapport Pédagogique et Communication, Paris, Mouton \& Co, The Hague, École Pratiques des Hautes Études, 39-69 
BOURDIEU P. \& PASSERON J.-C. 1970 La reproduction. Éléments pour une théorie du système d'enseignement, Paris, Minuit

CORTESÃO L. \& STOER S.R. 1997 "Investigação-Acção e a produção de conhecimento no âmbito de uma formação de professores para a educação inter/multicultural", Educação Sociedade e Culturas, 7, 7-28

DEROUET J-L. 1992 École et Justice. De l'égalité des chances aux compromis locaux?, Paris, Métailié

EISENSTADT S.N. 1991 A Dinâmica das Civilizações -tradição e modernidade, Lisboa, Edições Cosmos

ENGUITA M. 1996 "Escola e Etnicidade : o caso dos ciganos", Educação, Sociedade e Culturas, 6, 5-22

GIDDENS A. 1992 As consequências da modernidade, Oeiras, Celta Editora

GIDDENS A 1995 Transformações da intimidade. Sexualidade, Amor e Erotismo nas Sociedades Modernas, Oeiras, Celta

GIROUX H. 1986 Teoria crítica e resistência em educação. Para além das teorias de reprodução, Petrópolis, Editora Vozes

GIROUX H. 1997 Os Professores como intelectuais. Rumo a uma pedagogia crítica da aprendizagem, Porto Alegre, Editora Artes Médicas Sul

LIVET P. 1993 Théorie de l'action et conventions, in La théorie de l'action. Le sujet pratique en débat, Ladrière P., Pharo P., Quéré L. (coord.) Paris, CNRS Éditions, 291-318

MARTUCCELLI D. 1999 Sociologies de la modernité. L'itinéraire du XXe siècle, Paris, Gallimard

NISBET R. A. 1993 La tradition sociologique, Paris, Quadrige/PUF

NÓVOA, A. 1991a "O passado e o presente dos professores", in Noóvoa A. (org.) Profissão Professor, Porto, Porto Editora, 9-32

NÓVOA, A. 1991b “A formação contínua entre a pessoa-professor e a organização-escola”, Inovação, 4-1, 63-76

QUEIROZ J-M. 1995 L'École et ses sociologies, Paris, Nathan-Université

RESENDE J. M. 2001a O Engrandecimento de uma profissão. Os professores do Ensino Secundário Público no Estado Novo, Lisboa, Tese de Doutoramento em Sociologia, Faculdade de Ciências Sociais e Humanas da Universidade Nova de Lisboa

RESENDE J. M. 2001b “Individualidade, Denúncia e Modernidade : o sentido de justiças de um professor com a identidade magoada -o caso singular de uma denúncia pública no Estado Novo", Forum Sociológico, 5/6 (nova série), 101-127

RESENDE J. M. \& VIEIRA M. M. 2000 The multicultural issue in Portuguese schools seeking justice or another morality ?, in Christopher Day, Dolf van Veen (eds) Educacional Research in Europe-Yearbook 2000, Louvain \& Apeldoorn, Garant Publishers \& EERA, 203-218

RESENDE J. M. \& VIEIRA, M. M. 2002 "As cores da escola : concepções de Justiça nos discursos sobre a multiculturalidade na escola portuguesa", Actas do IV Congresso Português de Sociologia, Coimbra, APS, Acta 053 pdf, 1-20

SANTOS B. S. 1999 "Porque é tão difícil construir uma teoria crítica ?", Travessias, 1, 21 38 
SERRES M. 1996 Diálogo sobre a Ciência, a Cultura e o Tempo. Conversas com Bruno Latour, Lisboa, Instituto Piaget

STOER S. R. 1994 "Construindo a Escola Democrática através do campo de recontextualização pedagógica”, Educação, Sociedade e Culturas, 1, 7-27

STOER S. R., ARAÚJO H. 1991 Educação e Democracia num país semiperiférico (no contexto europeu), in STOER S. R. (org.), Educação, Ciências Sociais e Realidade Portuguesa, Porto, Edições Afrontamento, 205-230

STOER S. R. \& CORTESÃO L. 1999 "Levantando a Pedra", Da Pedagogia Inter/Multicultural às Políticas Educativas numa Época de Transnacionalização, Porto, Edições Afrontamento

TAYLOR C., APPIAH K. A., HABERMAS J., ROCKEFELLER S. C., WALZER M. \& WOLF S. 1998 Multiculturalismo. Examinando a política de reconhecimento, Lisboa, Instituto Piaget

THÉVENOT L. 1993 Agir avec d'autres. Conventions et objets dans l'action coordonnée, in Ladrière P., Pharo P., Quéré L. (coord.) La théorie de l'action. Le sujet pratique en débat, Paris, CNRS Éditions, 275-289

VIEIRA M. 2001 "Ensino Superior e Modernidade”, Forum Sociológico, 5/6, 169-184

VIEIRA M.-M., PINTASSILGO J. \& PORTUGAL E MELO B. (coords.) 2002 Actas do Colóquio "Democratização escolar: intenções e apropriações", Lisboa, Centro de Investigação em Educação da Faculdade de Ciências da Universidade de Lisboa, 143-172

VINCENT G. 1994 Forme scolaire et modèle républicain, in Vincent G. (dir.) L'éducation prisonnière de la forme scolaire ?, Lyon, PUL, 207-227

WAGNER P. 1996 Liberté et Discipline. Les deux crises de la modernité, Paris, Métailié 